Pacific Journal of Mathematics

POSITIVE LINEAR FUNCTIONS, INTEGRATION, AND 


\title{
POSITIVE LINEAR FUNCTIONS, INTEGRATION, AND CHOQUET'S THEOREM
}

\author{
Richard C. Metzler
}

The study of extensions of positive linear functions leads, in this paper, to a generalized and unified treatment of the Riemann, Lebesgue, Daniell, and Bourbaki integrals and of the Choquet-Bishop-deLeeuw integral representation theorem.

Let $\alpha$ be a positive linear function mapping from a subspace of an ordered vector space to another ordered vector space. Generalizing the process of extending the definition of an integral from some space of "simple" functions (e.g., continuous functions or step functions) to a larger space of integrable functions it is shown that extensions of $\alpha$ exist which are positive and linear and preserve a certain approximation property. In many cases of interest there is exactly one such extension which is maximal; in particular, this holds true for the generalizations of the familiar integrals of analysis. Different choices of approximating properties lead to different "integrals." With additional completeness assumptions on domain, range and function it is shown that the approximation property which leads to the Lebesgue integral in the function case gives an extension for which the generalizations of the usual convergence theorems hold. In the case when $\alpha$ is defined on the space of continuous functions on a compact Hausdorff space the correct choice of approximating property gives extensions which are measures supported by the Choquet boundary; this is the ChoquetBishop-de Leeuw theorem. Integrals with values in a locally convex space are treated by using an order determined by the topology of the space.

McShane in [7] and Alfsen in [1] have studied very general versions of integration. The treatment presented here differs in that we are concerned with showing how different integrals arise uniquely as maximal positive linear extensions. The idea that this is a natural and useful approach is reinforced by the fact that the Choquet theorem and also the Hahn-Banach theorem (see [6, p. 21]) appear as easy consequences.

1. Existence of positive linear $\boldsymbol{W}$-extensions. An ordered vector space is a vector space $V$ over the reals with an order relation " $\leqq$ " such that $x \leqq y$ implies $x+z \leqq y+z$ for all $z$ in $V$ and $r x \leqq r y$ for all nonnegative numbers $r$. If the requirement of antisymmetry is omitted from the order relation, $V$ is said to be a preordered vector space. In this case the set $V^{+}=\{x \in V: x \geqq 0\}$ forms a wedge in 
$V$ (i.e. $V^{+}+V^{+} \subset V^{+}$and $r V^{+} \subset V^{+}$for $r \geqq 0$ ) while if $V$ is an ordered vector space $V^{+}$satisfies the additional requirement $V^{+} \cap\left(-V^{+}\right)=\{0\}$ (i.e. $V^{+}$is a cone). An ordered vector space is said to be Dedekind complete (resp. monotone Dedekind complete) if every upper bounded subset (resp. increasingly directed upper bounded subset) has a least upper bound. A linear function $\alpha$ from $V$ to another preordered vector space $Y$ is said to be positive if $x \leqq y$ in $V$ implies $\alpha(x) \leqq \alpha(y)$ in $Y$. In the following $R$ will always designate the real numbers.

An ordered vector space $V$ is said to be a vector lattice if, for every pair of elments $u$ and $v$ in $V, \sup \{u, v\}$ (written $u \vee v$ ) and $\inf \{u, v\}$ (written $u \wedge v$ ) both exist in $V$. We denote $u \vee 0$ by $u^{+}$and $(-u) \vee 0$ by $u^{-}$and $u \vee(-u)$ by $|u|$ and note that $(u \vee v)+w=(u+w) \vee(v+w)$ and $(u \wedge v)+w=(u+w) \wedge(v+w)$. A subset $S$ of a vector lattice $V$ is said to be a sublattice of $V$ if, for every $u, v$ in $S$, we have $(u \vee v)$ and $(u \wedge v)$ in $S$ also.

For brevity we will write $z \leqq \alpha\left(g-V^{+}\right)$instead of $z \leqq y$ for all $y \in \alpha\left(g-V^{+}\right)$and $\sup \alpha\left(\left(g-V^{+}\right) \cap(-W)\right)$ will be written in place of $\sup \{\alpha(h): g \geqq h \in(-W) \cap d m n \alpha\}$.

Many times in the theory of integration we wish to be able to approximate the value of our integral by its value on some special class of elements (e.g. step functions, continuous functions, lower semicontinuous functions, etc.). We generalize this in the following way: Let $\alpha$ be a positive linear function from a subspace $G$ of a preordered vector space $V$ to an ordered vector space $Y$. Given a wedge $W \subset V$ we say that $\alpha$ is $W$-approximated if $W \subset G+V^{+}$and, for all $g \in G$, we have:

$$
\sup \alpha\left(\left(g-V^{+}\right) \cap(-W)\right) \text { exists in } Y \text { and equals } \alpha(g) \text {. }
$$

The first problem of integration can be viewed as that of finding an extension of a simple integral which is still positive and linear and which preserves some approximation property. In this section we are concerned with the existence, in our abstract situation, of extensions which are positive and linear and which preserve the $W$-approximation property.

THEOREM 1.1. Let $\alpha$ be a positive linear function from a subspace $G$ of a preordered vector space $V$ to an ordered vector space $Y$. Let $W$ be a wedge in $V$ such that $\alpha$ is $W$-approximated. Then $\alpha$ can be extended to a positive linear function $\hat{\alpha}$ such that $\hat{\alpha}$ is $W$-approximated and such that $\hat{\alpha}$ is maximal with respect to positive, linear, $W$ approximated extensions.

Proof. Let $M$ be the set of all positive linear $W$-approximated functions mapping from a subspace of $V$ into $Y$ and let $M$ be partially 
ordered by inclusion (as sets of ordered pairs). An application of Zorn's lemma gives the result.

An extension with the properties described above will be called a maximal W-extension.

Given a positive linear function $\alpha$ from a subspace $G$ of a preordered vector space $V$ to an ordered vector space $Y$ we define a new function $\underline{\alpha}$ by: $\underline{\alpha}(f)=\sup \alpha\left(f-V^{+}\right)$for all $f \in G+V^{+}$such that the sup exists in $Y$. We define $\bar{\alpha}$ in the obvious dual fashion. It is not hard to see that $f \in d m n \underline{\alpha}$ if and only if $-f \in d m n \bar{\alpha}$ and in this case $\bar{\alpha}(-f)=-\underline{\alpha}(f)$. If $\beta$ is any positive extension of $\alpha$ it is clear that $\underline{\alpha}(f) \leqq \beta(f)$ (and $\beta(f) \leqq \bar{\alpha}(f)$ ) wherever both sides of the inequality are defined. If $Y$ is Dedekind complete then $(d m n \underline{\alpha}) \cap(d m n \bar{\alpha})=$ $\left(G+V^{+}\right) \cap\left(G-V^{+}\right)$(i.e. both the "upper integral" and the "lower integral" are always defined on " $G$-bounded elements").

LEMMA 1.2. Let $\alpha$ be a positive linear function from a subspace $G$ of a preordered vector space $V$ to an ordered vector space $Y$. Let $W$ be $a$ wedge in $V$ such that $\alpha$ is $W$-approximated and suppose that $f \in d m n \underline{\alpha}$. If we define $\alpha_{1}$ on $G+R f$ by $\alpha_{1}(g+r f)=\alpha(g)+r \underline{\alpha}(f)$ then $\alpha_{1}$ is linear and positive and, for $r>0, \sup \alpha\left(\left(g+r f-V^{+}\right) \cap(-W)\right)$ exists and equals $\alpha_{1}(g+r f)$.

Proof. It is clear that $\alpha_{1}$ is a linear extension of $\alpha$ and it is easily shown that $\alpha_{1}$ is positive [6].

For the last property let $h_{1} \in(-W) \cap G$ be such that $g \geqq h_{1}$ and let $h_{2} \in G$ be such that $f \geqq h_{2}$. Now let $h_{3}$ in $(-W) \cap G$ be such that $h_{3} \leqq h_{2}$. Then $g+r f \geqq h_{1}+r h_{3} \in(-W) \cap G$ so if $y \in Y$ is any upper bound for $\alpha\left(\left(g+r f-V^{+}\right) \cap(-W)\right)$ then $y \geqq \alpha\left(h_{1}+r h_{3}\right)$. This shows that $y-r \alpha\left(h_{3}\right) \geqq \alpha\left(h_{1}\right)$ for all $h_{1} \in(-W) \cap G$ such that $h_{1} \leqq g$. Hence $y-r \alpha\left(h_{3}\right) \geqq \sup \alpha\left(\left(g-V^{+}\right) \cap(-W)\right)=\alpha(g)$ by $W$-approximation. Thus $(1 / r)(y-\alpha(g)) \geqq \alpha\left(h_{3}\right)$ for all $h_{3} \in(-W) \cap G$ such that $h_{3} \leqq$ $h_{2}$. Again $W$-approximation shows that $(1 / r)(y-\alpha(g)) \geqq \alpha\left(h_{2}\right)$ and since this is true for all $h_{2} \in G$ such that $h_{2} \leqq f$ we have $(1 / r)$ $(y-\alpha(g)) \geqq \underline{\alpha}(f)$. Thus $y \geqq \alpha_{1}(g+r f)$. This shows that $\alpha_{1}(g+r f)$ is smaller than any upper bound of $\alpha\left(\left(g+r f-V^{+}\right) \cap(-W)\right)$. Since $\alpha_{1}$ is positive this element clearly is an upper bound itself so it is the required least upper bound.

THEOREM 1.3. Let $\alpha$ be a positive linear function from a subspace $G$ of a preordered vector space $V$ to an ordered vector space $Y$. Let $W$ be a wedge in $V$ such that $\alpha$ is $W$-approximated and suppose that $f \in d m n \underline{\alpha}$. If $f \in W$ then there is a maximal $W$-extension which takes the value $\underline{\alpha}(f)$ on $f . \quad$ Alternatively if $f \in d m n \bar{\alpha}$ and $\bar{\alpha}(f)=\underline{\alpha}(f)$ then all maximal $W$-extensions are defined on $f$ and take the value $\underline{\alpha}(f)$ on $f$. 
Proof. As before we define $\alpha_{1}$ on $G+R f$ by $\alpha_{1}(g+r f)=$ $\alpha(g)+r \underline{\alpha}(f)$. The lemma shows that $\alpha_{1}$ is linear and positive and that $\alpha_{1}$ is $W$-approximated on $g+r f$ for $r>0$.

If $f \in W$ and $r \leqq 0$ in $g+r f$ we note first that $\alpha_{1}(g+r f)$ is an upper bound for $\alpha_{1}\left(\left(g+r f-V^{+}\right) \cap(-W)\right)$ because $\alpha_{1}$ is positive. Let $y \in Y$ be any other bound for this set. Then, since $r f \in-W$ and since $-W$ is a wedge, for any $h \in(-W) \cap G$ with $h \leqq g$ we have $g+r f \geqq$ $h+r f \in(-W) \cap(G+R f)$. Therefore:

$$
\begin{aligned}
y \geqq \sup \left\{\alpha_{1}(h+r f): g \geqq h \in(-W) \cap G\right\} \\
=\sup \alpha\left(\left(g-V^{+}\right) \cap(-W)\right)+\alpha_{1}(r f) \\
=\alpha(g)+\alpha_{1}(r f)=\alpha_{1}(g+r f) .
\end{aligned}
$$

So $\alpha_{1}(g+r f)$ is the least upper bound. Then $\alpha_{1}$ is $W$ approximated and any maximal $W$-extension of $\alpha_{1}$ is of the required sort.

To prove the last statement suppose that $f \in d m n \bar{\alpha}$ and $\bar{\alpha}(f)=$ $\underline{\alpha}(f)$. Let $\hat{\alpha}$ be any maximal $W$-extension of $\alpha$ and note first that $\underline{\alpha}(f) \leqq(\underline{\hat{\alpha}})(f) \leqq(\overline{\hat{\alpha}})(f) \leqq \bar{\alpha}(f)$. If we define $\tilde{\alpha}$ on $d m n \hat{\alpha}+R f$ by $\tilde{\alpha}(h+r f)=\hat{\alpha}(h)+r(\underline{\hat{\alpha}})(f)$ then the lemma shows that $\tilde{\alpha}$ is linear and positive and that $\tilde{\alpha}$ is $W$-approximated on $g+r f$ for $g \in d m n \hat{\alpha}$ and $r>0$. If we note that $(\underline{\hat{\alpha}})(-f)=-\tilde{\alpha}(f)$ and apply the lemma to $-f$ we find that the $W$-approximation property holds also for $r<0$. Thus $\tilde{\alpha}$ is $W$-approximated and maximality shows that $\tilde{\alpha}=\hat{\alpha}$. Finally the inequality $\underline{\alpha}(f) \leqq \hat{\alpha}(f) \leqq \bar{\alpha}(f)$ gives the required uniqueness.

2. Unique extensions. For applications a maximal extension is often too "large" to be useful. It is not possible in general to determine what the value of the extension is on a given element since the extension is not constructed. Another drawback is the fact that different extensions may give different values on the same element. However, in the pleasant situation when there is exactly one maximal $W$-extension these difficulties disappear. In our approach to integration all integrals will be defined as the unique maximal, positive, linear, $W$-approximated extension. The following theorem characterizes the domain of a unique maximal $W$-extension and gives necessary and sufficient conditions for uniqueness.

THEOREM 2.1. Let $\alpha$ be a positive linear function from a subspace $G$ of a preordered vector space $V$ to an ordered vector space $Y$. Let $W$ be a wedge in $V$ such that $\alpha$ is $W$-approximated. Then there is exactly one maximal $W$-extension of $\alpha$ if we have the following condition:

(*) Let $f \in W, g \in-W$ and suppose that $f \geqq g$. Then, if $y$ and $z$ in $Y$ are such that $y \geqq \alpha\left(f-V^{+}\right)$and $z \leqq \alpha\left(g+V^{+}\right)$, we have $y \geqq z$. 
In this case if we designate this unique maximal $W$-extension by $\hat{\alpha}$ we have $\hat{\alpha} \cap(W \times Y)=\underline{\alpha} \cap(W \times Y)$. For any $v \in V$, we have $v \in$ dmn $\hat{\alpha}$ if and only if inf $\left.\underline{\alpha}\left(\left(v+V^{+}\right) \cap W\right)\right)=\sup \bar{\alpha}\left(\left(v-V^{+}\right) \cap(-W)\right)$.

If $Y$ is Dedekind complete, then $(*)$ is also a necessary condition for uniqueness.

Proof. Let $\hat{\alpha}$ be any maximal $W$-extension of $\alpha$ and choose an $f \in W$. If $A, B$, and $C$ denote the sets of upper bounds in $Y$ of $\alpha\left(f-V^{+}\right), \quad \hat{\alpha}\left(\left(f-V^{+}\right) \cap(-W)\right), \quad$ and $\hat{\alpha}\left(f-V^{+}\right)$respectively, $W$ approximation shows that $A \supset B$ and $B=C$. But if $y \in A$ and $z=$ $\hat{\alpha}(g)$ for some $g \in\left(f-V^{+}\right) \cap(-W)$ then, since $\hat{\alpha}(g) \leqq \alpha\left(g+V^{+}\right)$, we conclude by condition $(*)$ that $y \geqq \hat{\alpha}(g)$. Hence $y \in B$ and we have equality of the three sets of upper bounds. Thus $f \in d m n \underline{\alpha} \cap W$ if and only if $f \in d m n(\underline{\hat{\alpha}}) \cap W$. By Theorem 1.3 and maximality of $\hat{\alpha}$ we conclude that $f \in d m n \underline{\alpha} \cap W$ if and only if $f \in d m n \hat{\alpha} \cap W$. In this case $\hat{\alpha}(f)$ is the least upper bound of $\hat{\alpha}\left(\left(f-V^{+}\right) \cap(-W)\right)$ by $W$ approximation and, since $A=B$ above, we see that $\hat{\alpha}(f)=$ $\underline{\alpha}(f)$. Hence $\hat{\alpha} \cap(W \times Y)=\underline{\alpha} \cap(W \times Y)$.

Now if $f$ is any element of $d m n \hat{\alpha}$ then $W$-approximation shows that $\hat{\alpha}(f)=\inf \hat{\alpha}\left(\left(f+V^{+}\right) \cap W\right)=\inf \underline{\alpha}\left(\left(f+V^{+}\right) \cap W\right)$. Similarly we see that $\hat{\alpha}(f)=\sup \bar{\alpha}\left(\left(f-V^{+}\right) \cap(-W)\right)$. On the other hand if we are given that an $f \in V$ satisfies the equality inf $\underline{\alpha}\left(\left(f+V^{+}\right) \cap W\right)=$ $\sup \bar{\alpha}\left(\left(f-V^{+}\right) \cap(-W)\right)$ then $\overline{(\hat{\alpha})}(f)=(\underline{\hat{\alpha}})(f)$ so, by Theorem 1.3 and maximality of $\hat{\alpha}$, we have $f \in d m n \hat{\alpha}$ and $\hat{\alpha}(f)$ is equal to the common value. Thus there is exactly one maximal $W$-extension.

Finally, let $Y$ be Dedekind complete and suppose that there is exactly one maximal $W$-extension $\hat{\alpha}$. Let $f \in W, g \in-W$ and suppose that $f \geqq g$. If $y$ and $z$ in $Y$ are such that $y \geqq \alpha\left(f-V^{+}\right)$and $z \leqq \alpha\left(g+V^{+}\right)$then $f$ is in $d m n \underline{\alpha}$ and $g$ is in $d m n \bar{\alpha}$. By Theorem 1.3 and uniqueness of $\alpha$ we have $f$ and $g$ in $d m n \hat{\alpha}$ and $\underline{\alpha}(f)=\hat{\alpha}(f) \geqq \hat{\alpha}(g)=$ $\bar{\alpha}(g)$. Since $y \geqq \underline{\alpha}(f)$ and $z \leqq \bar{\alpha}(g)$ we see that condition $(*)$ is fulfilled.

In the future if there is exactly one maximal $W$-extension of a function $\alpha$ we will designate it $\alpha_{w}$.

With this approach to abstract integration it is automatic that the set of "integrable elements" is a subspace. In the next theorem we investigate under what circumstances the subspace is a sublattice.

THEOREM 2.2. Let $\alpha$ be a positive linear function from a sublattice $G$ of a vector lattice $V$ to a monotone Dedekind complete space $Y$. If $W$ is a wedge in $V$ which is a sublattice of $V$ and which is such that $\alpha$ has exactly one maximal $W$-extension, then $d m n \alpha_{W}$ is a sublattice of $V$. 
Proof. If $f$ and $g$ are in $W \cap d m n \alpha_{W}$ then $f \wedge g \in W \cap d m n \underline{\alpha}$ by the assumption on $W$ and because $Y$ is monotone Dedekind complete and $G$ is a sublattice. By Theorem $2.1, f \wedge g$ is in $W \cap$ $d m n \alpha_{W}$. Furthermore we can show that $f^{+}$is in $d m n \alpha_{W} \cap W$. In fact, if we choose $c$ in $G$ such that $f \geqq c$, we have $f-(c \wedge 0)=(f-c) \vee f \geqq$ $f^{+}$, so $f^{+}$is in dmn $\underline{\alpha}$ by monotone Dedekind completeness of $Y$.

Now if $v$ is in $d m n \alpha_{W}$ and $h$ and $g$ are such that $h \leqq v \leqq g$ with $g$ and $-h$ in $W \cap d m n \alpha_{W}$, then $h \wedge 0 \leqq v \wedge 0 \leqq g \wedge 0$. Furthermore

$$
g \wedge 0-h \wedge 0=-g^{-}+h^{-} \leqq-g^{-}+h^{-}+g^{+}-h^{+}=g-h .
$$

Then

$$
\begin{aligned}
& 0 \leqq \\
& \quad \inf \left\{\alpha_{W}(g \wedge 0): v \leqq g \in W \cap d m n \alpha_{W}\right\} \\
& \quad-\sup \left\{\alpha_{W}(h \wedge 0): v \geqq h \in(-W) \cap d m n \alpha_{W}\right\} \\
& =\inf \left\{\alpha_{W}(g \wedge 0)-\alpha_{W}(h \wedge 0): v \leqq g \in W \cap d m n \alpha_{W},\right. \\
& \left.\qquad v \geqq h \in(-W) \cap d m n \alpha_{W}\right\} \\
& \leqq \\
& \inf \left\{\alpha_{W}(g-h): v \leqq g \in W \cap d m n \alpha_{W}, v \geqq h \in(-W) \cap d m n \alpha_{W}\right\} \\
& =\alpha_{W}(v)-\alpha_{W}(v)=0 \text { by } W \text {-approximation. }
\end{aligned}
$$

(The existence of the required sups and infs above follows from the fact that $Y$ is monotone Dedekind complete and the fact that $W \cap d m n \alpha_{W}$ is closed under finite infs.) We conclude that $\left(\alpha_{W}\right)(v \wedge 0)=\overline{\left(\alpha_{W}\right)}(v \wedge 0)$ so $v \wedge 0$ is in $d m n \alpha_{W}$ by Theorem 1.3 and maximality. Since $a \wedge b=$ $(a-b) \wedge 0+b$ for any $a$ and $b$ in $V$ it follows easily that $d m n \alpha_{W}$ is a sublattice.

3. The Riemann extension. In this section we will consider a generalization of the Darboux approach to the Riemann integral.

THEOREM 3.1. Let $\alpha$ be a positive linear function from a subspace $G$ of a preordered vector space $V$ to an ordered vector space $Y$. Then there is exactly one maximal G-extension, $\alpha_{G}$, and $\alpha_{G}=\underline{\alpha} \cap \bar{\alpha}$.

Proof. It is clear that $\alpha$ is $G$-approximated and that condition (*) of Theorem 2.1 is satisfied, so there is a unique maximal $G$ extension. Furthermore the equality condition on the domain of $\alpha_{G}$ in that theorem reduces in this case to $\alpha_{G}=\underline{\alpha} \cap \bar{\alpha}$.

The function $\alpha_{G}$ will be referred to as the Riemann extension of $\alpha$. The reason for this name is apparent from the following application. Let $V=R^{R}$ and let $Y=R$. Suppose $G$ is the subspace 
of $V$ composed of step functions which vanish outside of a bounded interval and suppose that $\alpha$ is the usual "signed area" functional. Then for any bounded function $f$ which vanishes outside of a bounded interval we see that $\underline{\alpha}(f)$ is the lower Darboux integral of $f$ and $\bar{\alpha}(f)$ is the upper Darboux integral of $f$. The previous theorem then shows that the Riemann extension of $\alpha$ gives the usual Riemann integral.

THEOREM 3.2. Let $\hat{\alpha}$ be any maximal $W$-extension of a positive linear function $\alpha$ from a subspace $G$ of a preordered vector space $V$ to an ordered vector space $Y$. Then $\hat{\alpha}$ is equal to its Riemann extension.

Proof. If $f \in V$ is such that $(\underline{\hat{\alpha}})(f)=\overline{(\hat{\alpha})}(f)$ then Theorem 1.3 shows that there is a maximal $W$-extension of $\hat{\alpha}$ defined on $f$. Maximality of $\hat{\alpha}$ and the previous theorem complete the proof.

4. The unbounded Riemann extension. The Riemann extension has the weakness that it is defined only for elements that are bounded above and below by elements of $G$. In this section we define an extension with a larger domain. This extension generalizes the improper Riemann integeral and will also be used to obtain our generalizations of the Lebesgue integral.

THEOREM 4.1. Let $\alpha$ be a positive linear function from a sublattice $G$ of a vector lattice $V$ to an ordered vector space $Y$. Let $U=$ $\left\{f \in G+V^{+}: f \wedge g \in G\right.$ for each $\left.g \in G\right\}$. Then $U$ is a wedge and $\alpha$ is $U$-approximated. Furthermore there is exactly one maximal $U$ extension, $\alpha_{U}$.

Proof. To see that $U$ is a wedge suppose first that $f, h \in U^{+}$and let $g$ be any element of $G^{+}$. Then we have:

$$
\begin{aligned}
(f+h) \wedge g & =(f+h) \wedge((f+g) \wedge(h+g) \wedge(g+g) \wedge g) \\
& =((f \wedge g)+h \wedge g)) \wedge g \in G .
\end{aligned}
$$

Now if $f$ and $h$ are any elements of $U$ and $g$ is any element of $G$ choose $p$ in $G^{+}$such that $f+p \geqq 0, h+p \geqq 0$, and $g+2 p \geqq 0$. Since $(f+p) \wedge q=f \wedge(q-p)+p$ for all $q$ in $G$ we see that $f+p$ (and similarly $h+p$ ) is in $U^{+}$. Consequently:

$$
(f+h) \wedge g=((f+p)+(h+p)) \wedge(g+2 p)-2 p \in G .
$$

Since it is easy to see that $r U \subset U$ for all nonnegative real numbers $r$, we conclude that $U$ is a wedge. 
Since $G \subset U$ it is clear that $\alpha$ is $U$-approximated. Suppose $f$ is in $U$ (in fact we need only assume $f$ is in $G+V^{+}$) and suppose $f \geqq$ $g \in(-U)$. Choosing $h \in G$ such that $f \geqq h$ we have $f \geqq g \vee h \geqq$ $g$. Since $g \vee h \in G$ by definition of $U$ we see that $y \geqq \alpha\left(f-V^{+}\right)$and $z \leqq \alpha\left(g+V^{+}\right)$implies $y \geqq \alpha(g \vee h) \geqq z$ so condition $(*)$ of Theorem 2.1 is satisfied. (Note that if $f \in\left(G+V^{+}\right) \cap d m n \alpha_{U}$ then this argument shows that $\alpha_{U}(f)=\underline{\alpha}(f)$.)

The unique $U$-extension of this theorem will be called the unbounded Riemann extension. The next theorem gives an alternate characterization of the domain of this extension which we will use to show that we have a generalization of one definition of the improper Riemann integral.

THEOREM 4.2. Let $\alpha$ be a positive linear function from a sublattice $G$ of a vector lattice $V$ to a monotone Dedekind complete space $Y$. Then $d m n \alpha_{U}$ is a sublattice of $V$ and $\alpha_{U}(v)=\underline{\alpha}\left(v^{+}\right)-\underline{\alpha}\left(v^{-}\right)$for all $v$ in dmn $\alpha_{U}$. If, furthermore, $\alpha$ is equal to its own Riemann extension, then $v$ is in $d m n \alpha_{U}$ if and only if $v^{+}$and $v^{-}$are in $U \cap d m n \alpha_{U}$.

Proof. It is easy to see that $U$ is a sublattice of $V$ so Theorem 2.2 shows that $d m n \alpha_{U}$ is a sublattice of $V$. If $v \in d m n \alpha_{U}$ then $v^{+} \in$ $\left(G+V^{+}\right) \cap d m n \alpha_{U}$ and the remark in the last line of the proof of the previous theorem shows that $\alpha_{U}\left(v^{+}\right)=\underline{\alpha}\left(v^{+}\right)$. The same argument for $-v$ allows us to conclude that $\alpha_{U}\left(v^{-}\right)=\underline{\alpha}\left(v^{-}\right)$.

To prove the last statement of the theorem assume that $\alpha$ is equal to its own Riemann extension. Clearly if $v^{+}$and $v^{-}$are in $U \cap d m n \underline{\alpha}$ then $v$ is in $d m n \alpha_{U}$. For the converse let $v$ be any element of $d m n \alpha_{U}$. Then, for any $g \in G, v^{+} \wedge g$ is an element of $d m n \alpha_{U}$ which is bounded above and below by elements of $G$. But then $U$ approximation implies that $\underline{\alpha}$ and $\bar{\alpha}$ coincide on $v^{+} \wedge g$. Since $g$ was arbitrary $v^{+}$is in $U$.

This theorem shows that when $\alpha$ is the usual Riemann integral $\alpha_{U}$ is the improper Riemann integral as defined by de la Vallee Poussin.

5. Sequential and non-sequential extensions. In this section we will develop two new extensions which will have useful convergence properties. The first extension generalizes the $\sigma$-algebra approach to integration and the second will be used to obtain our generalization of the integral of Bourbaki. In order to have an extension with desirable convergence properties the original function must preserve limits in the following way. 
Let $\alpha$ be a positive linear function from a subspace $G$ of a preordered vector space $V$ to an ordered vector space $Y$. Then $\alpha$ is said to have the Daniell property if, for every lower bounded decreasing sequence $\left\{f_{n}\right\}$ in $G$ with only negative lower bounds in $V$, the sequence $\left\{\alpha\left(f_{n}\right)\right\}$ in $Y$ has only negative lower bounds or none at all. We will call such a function a Daniell function.

Given a subspace $G$ of an ordered vector space $V$ we designate by $S(G)$ (just written $S$ when no confusion is possible) the set of elements of $V$ which are sups of increasing sequences from $G$. It is easy to see that $S$ is a wedge and that $\alpha$ is $S$-approximated for any positive linear $\alpha$ defined on $G$.

THEOREM 5.1. Let $\alpha$ be a Daniell function from a subspace $G$ of an ordered vector space $V$ to an ordered vector space $Y$. Then there is exactly one maximal $S$-extension of $\alpha$. If $G$ contains a sequence $\left\{f_{n}\right\}$ such that $f_{n} \uparrow_{n=1,2 \ldots} f \in S$ then $f \in d m n \underline{\alpha}$ if and only if $\sup _{n} \alpha\left(f_{n}\right)$ exists in $Y$. In this case $f \in d m n \alpha_{s}$ and $\alpha_{S}(f)=\underline{\alpha}(f)=\sup _{n} \alpha\left(f_{n}\right)$.

Note. When we write $f_{n} \underset{n=1,2, \cdots}{f} f$ we mean that $\left\{f_{n}\right\}$ is an increasing sequence and $f=\sup _{n} f_{n}$.

Proof. Let $f \in S, g \in-S$ and $f \geqq g$ and suppose that $y$ and $z$ in $Y$ are such that $y \geqq \alpha\left(f-V^{+}\right)$and $z \leqq \alpha\left(g+V^{+}\right)$. By definition of $S$ there are sequences $\left\{g_{n}\right\}$ and $\left\{f_{n}\right\}$ in $G$ such that $g_{n} \underset{n=1,2, \ldots}{\downarrow} g$ and $f_{n} \underset{n=1,2, \cdots}{ } f$. Then the fact that $f \geqq g$ shows that the lower bounded decreasing sequence $\left\{g_{n}-f_{n}\right\}$ has only negative lower bounds. Since $z-y$ is a lower bound of $\left\{\alpha\left(g_{n}-f_{n}\right)\right\}$ the Daniell property shows that $y \geqq z$. By Theorem 2.1 we conclude that there is a unique maximal $S$-extension $\alpha_{S}$ and that $\alpha_{S} \cap(S \times Y)=\underline{\alpha} \cap(S \times Y)$.

To prove the next statement we need to show that when $G \ni$ $f_{n} \underset{n=1,2, \cdots}{ } f \in S$ then the sequence $\left\{\alpha\left(f_{n}\right)\right\}$ and the set $\alpha\left(f-V^{+}\right)$have the same set of upper bounds. Clearly if $y \geqq \alpha(g)$ for all $g$ in $G$ such that $g \leqq f$ then $y \geqq \alpha\left(f_{n}\right)$ for all $n=1,2, \cdots$. On the other hand if $y \geqq \alpha\left(f_{n}\right)$ for all $n=1,2, \cdots$ let $g \in G$ be such that $g \leqq f$. Then $\left\{g-f_{n}\right\}$ is a sequence which is decreasing and has only negative lower bounds in $V$. Thus $\alpha(g)-y \leqq \alpha(g)-\alpha\left(f_{n}\right)$ for all $n=1,2, \cdots$ implies $\alpha(g) \leqq$ $y$. This shows the equality of the two sets of upper bounds and we conclude that $f \in d m n \underline{\alpha}$ if and only if $\sup _{n} \alpha\left(f_{n}\right)$ exists and, in this case, $\underline{\alpha}(f)=\sup _{n} \alpha\left(f_{n}\right)$.

THEOREM 5.2. Let $\alpha$ be a Daniell function from a sublattice $G$ of a vector lattice $V$ to an ordered vector space $Y$. Let $f=\sup _{n} f_{n}$ where $\left\{f_{n}\right\}$ 
is an increasing sequence in $S \cap d m n \alpha_{S}$. Then $\sup _{n} \alpha_{S}\left(f_{n}\right)$ exists in $Y$ if and only if $f \in d m n \underline{\alpha}$. In this case $f \in d m n \alpha_{s}$ and $\sup _{n} \alpha_{S}\left(f_{n}\right)=\underline{\alpha}(f)=$ $\alpha_{S}(f)$.

Proof. For each $n=1,2, \cdots$ there is a sequence $\left\{f_{n, m}\right\} \subset G$ such that $f_{n, m} \uparrow_{m=1,2, .} f_{n}$. If we define $h_{n}=f_{1, n} \vee \cdots \vee f_{n, n}$, then $h_{n} \in G$ for all $n=1,2, \cdots$ since $G$ is a sublattice, and $f=\sup _{n} h_{n}$. Since $\left\{h_{n}\right\}$ is an increasing sequence we see that $f \in S$.

To complete the proof we need only show that any upper bound of the sequence $\left\{\alpha_{S}\left(f_{n}\right)\right\}$ is an upper bound of $\alpha\left(f-V^{+}\right)$and vice versa. If $y \geqq \alpha(g)$ for all $g \in G$ such that $g \leqq f$ then $y \geqq \alpha\left(f_{n, m}\right)$ for all $n, m=$ $1,2, \cdots$. Hence $y \geqq \sup _{m} \alpha\left(f_{n, m}\right)=\alpha_{S}\left(f_{n}\right)$ for all $n=1,2, \cdots$. On the other hand if $y \geqq \alpha_{S}\left(f_{n}\right)$ for all $n=1,2, \cdots$ then, for $h_{n}=f_{1, n} \vee \cdots \vee f_{n, n}$, we have $y \geqq \alpha\left(h_{n}\right)$ for all $n=1,2, \cdots$. Then if $g \in G$ is such that $f \geqq g$ the lower bounded decreasing sequence $\left\{g-h_{n}\right\}$ has only negative lower bounds in $V$. Since $\alpha(g)-y \leqq \alpha(g)-\alpha\left(h_{n}\right)$ for all $n=1,2, \cdots$ we conclude $\alpha(g) \leqq y$.

We can apply the sequential extension in the familiar measuretheoretic approach to integration. Let $\Omega$ be a set and let $\mathscr{A}$ be a $\sigma$-algebra of subsets of $\Omega$. If $\mu$ is a measure on $\mathscr{A}$ we let $G_{\mu}$ be the linear sublattice of $R^{\Omega}$ composed of linear combinations of characteristic functions of sets of finite $\mu$-measure. If $\alpha$ is defined as the usual integral on $G_{\mu}$ it is a Daniell function [9, 10]. It can be easily seen that a positive $f$ is in $S\left(G_{\mu}\right)$ if and only if $f$ is a finite-valued measurable function in $G_{\mu}+V^{+}$which vanishes outside a countable union of sets of finite measure. Finally it can be shown that the functions in the domain of $\alpha_{S}$ are those finite-valued functions which are integrable by the completion [9; p. 211] of $\mu$. With this interpretation, Theorem 5.2 is seen to be the monotone convergence theorem for finite-valued, integrable, $\mu$-measurable functions.

Now we investigate the situation when our function $\alpha$ has a stronger convergence property. Let $\alpha$ be a positive linear function from a subspace $G$ of a preordered vector space $V$ to an ordered vector space $Y$. Then $\alpha$ is said to be a non-sequential Daniell function if, for every lower bounded decreasingly directed family $\left\{f_{\lambda}: \lambda \in \Lambda\right\} \subset G$ with only negative lower bounds in $V$, the family $\left\{\alpha\left(f_{\lambda}\right) ; \lambda \in \Lambda\right\} \subset Y$ has only negative lower bounds or none at all.

We designate by $N(G)$ (just written $N$ when no confusion is possible) the family of elements of $V$ which are sups of increasingly directed families from $G$. It is easy to see that $N$ is a wedge and that $\alpha$ is $N$-approximated for any positive linear $\alpha$ defined on $G$. If $\alpha$ is a non-sequential Daniell function, Theorems 5.1 and 5.2 still hold when $N$ is substituted everywhere for $S$ and increasingly directed families are 
substituted for increasingly directed sequences. The proofs are the obvious variants of the proofs in the sequential case.

When $\alpha$ is a non-sequential Daniell function we call the unique $N$-extension, $\alpha_{N}$, the non-sequential extension of $\alpha$. In order to use the results of $\S 4$ in the next section we need to know when $d m n \alpha_{S}$ and $d m n \alpha_{N}$ are sublattices.

Theorem 5.3. Let $\alpha$ be a Daniell (resp. non-sequential Daniell) function from a sublattice $G$ of a vector lattice $V$ to a monotone Dedekind complete space $Y$. Let $H=d m n \alpha_{S} \quad$ (resp. $\left.H=d m n \alpha_{N}\right)$. Then $H$ is a sublattice of $V$.

Proof. In light of Theorem 2.2 we need only show that $S$ (or $N$ in the non-sequential case) is a sublattice. This follows easily from the fact that $G$ is a sublattice.

6. Convergence theorems. In this section we define four different "generalized integrals" from vector lattices to vector lattices and prove the generalizations of the monotone convergence theorem, Fatou's lemma, and the dominated convergence theorem. In order to prove these results we must make additional assumptions about the domain and range. We assume that $V$ is a Dedekind $\sigma$-complete vector lattice (i.e. countable upper bounded families have a least upper bound). We also assume that, if $y \neq z$ in $Y$, then there is a real valued nonsequential Daniell function $\beta$ defined on $Y$ such that $\beta(y) \neq \beta(z)$. We say that $Y$ is a $Q$-space if it is monotone Dedekind complete and has this property. An example of a $Q$-space is furnished by the space of all real valued functions on any set in the usual pointwise order. Another example is the space of self-adjoint operators on a Hilbert space.

Theorem 6.1. Let $\alpha$ be a Daniell (resp. nonsequential Daniell) function from a sublattice $G$ of a Dedekind $\sigma$-complete vector lattice $V$ to a $Q$-space $Y$. Let $\beta=\alpha_{S}$ (resp. $\beta=\alpha_{N}$ ) and let $W=S$ (resp. $W=N)$. Then, if $h_{n} \underset{n=1,2, \cdots}{ } h \leqq g \in W \cap d m n \beta$ and if $h_{1} \in d m n \bar{\beta}$, we have $\sup _{n} \bar{\beta}\left(h_{n}\right)=\bar{\beta}(h)$.

Proof. By Theorem $5.3 d m n \beta$ is a sublattice of $V$. Hence, since $Y$ is monotone Dedekind complete, we see that $h$ and $h_{n}$ for all $n=1,2, \cdots$ are elements of $d m n \bar{\beta}$. Also $\sup _{n} \bar{\beta}\left(h_{n}\right)$ exists in $Y$ and is less than or equal to $\bar{\beta}(h)$. If these elements were not equal there would be a non-sequential Daniell function $D$ such that $D(\bar{\beta}(h)) \neq D\left(\sup _{n} \bar{\beta}\left(h_{n}\right)\right)$. We will show that this leads to a contradiction by adapting an argument appearing in [2; p. 110]. 
First note that if $y_{\lambda} \uparrow_{\lambda \in \Lambda} y$, then $D\left(y_{\lambda}\right) \uparrow_{\lambda \in \Lambda} D(y)$. This follows since $D(y)$ is an upper bound by positivity of $D$ and since $\left\{D\left(y-y_{\lambda}\right): \lambda \in \Lambda\right\}$ has only negative lower bounds in $R$. Thus $D(\bar{\beta}(h))=\inf \{D(\beta(f))$ : $h \leqq f \in d m n \beta\}$ and

$$
D\left(\sup _{n} \bar{\beta}\left(h_{n}\right)\right)=\sup _{n}\left\{\inf \left\{D(\beta(m)): h_{n} \leqq m \in d m n \beta\right\}\right\}
$$

By $W$-approximation, for each $n=1,2, \cdots$ we can choose $g_{n} \in W \cap d m n \beta \quad$ such that $g_{n} \geqq h_{n}$ and $D\left(\beta\left(g_{n}\right)\right)-\epsilon \cdot 2^{-n}<$ $D\left(\bar{\beta}\left(h_{n}\right)\right)$. We can also assume that $g_{n} \leqq g$ for all $n$ by taking $g_{n} \wedge g$ if necessary since $W$ is a sublattice of $V$. Now let $g_{n}^{\prime}=$ $g_{1} \vee \cdots \vee g_{n} \in W \cap d m n \beta$. If we note that $g_{n+1}^{\prime}+\left(g_{n}^{\prime} \wedge g_{n+1}\right)=$ $\left(g_{n}^{\prime} \vee g_{n+1}\right)+\left(g_{n}^{\prime} \wedge g_{n+1}\right)=g_{n}^{\prime}+g_{n+1}$ we have

$$
\begin{aligned}
D\left(\beta\left(g_{n+1}^{\prime}\right)\right) & =D\left(\beta\left(g_{n}^{\prime}\right)\right)+D\left(\beta\left(g_{n+1}\right)\right)-D\left(\beta\left(g_{n}^{\prime} \wedge g_{n+1}\right)\right) \\
& \leqq D\left(\beta\left(g_{n}^{\prime}\right)\right)+D\left(\beta\left(g_{n+1}\right)\right)-D\left(\bar{\beta}\left(h_{n}\right)\right) \\
& \leqq D\left(\beta\left(g_{n}^{\prime}\right)\right)+D\left(\bar{\beta}\left(h_{n+1}\right)\right)-D\left(\bar{\beta}\left(h_{n}\right)\right)+\epsilon \cdot 2^{-(n+1)} .
\end{aligned}
$$

Subtracting $D\left(\beta\left(g_{n}^{\prime}\right)\right)$ and adding we find $D\left(\beta\left(g_{n+1}^{\prime}\right)\right)<$ $D\left(\bar{\beta}\left(h_{n+1}\right)\right)+\epsilon$. Now $g_{n}^{\prime} \leqq g$ for all $n=1,2, \cdots$ implies that $g_{0}=\sup _{n} g_{n}^{\prime}$ exists in $V$ since $V$ is assumed Dedekind $\sigma$-complete. By Theorem 5.2 (or its nonsequential version) $g_{0} \in W \cap d m n \underline{\beta}$ and $\beta\left(g_{0}\right)=$ $\sup _{n} \beta\left(g_{n}^{\prime}\right)$. Clearly $g_{0} \geqq h$ so we see that $D(\bar{\beta}(h)) \leqq D\left(\beta\left(g_{0}\right)\right)=$ $\sup _{n} D\left(\beta\left(g_{n}^{\prime}\right)\right) \leqq D\left(\sup _{n} \bar{\beta}\left(h_{n}\right)\right)+\epsilon$.

Since $\epsilon$ was arbitrary we see that $D(\bar{\beta}(h)) \leqq D\left(\sup _{n} \bar{\beta}\left(h_{n}\right)\right)$. The other inequality follows by positivity so we have the desired contradiction.

Theorem 6.2. Let $\alpha$ be a Daniell (resp. nonsequential Daniell) function from a sublattice $G$ of a Dedekind $\sigma$-complete vector lattice $V$ to a Q-space $Y$. Let $\beta=\alpha_{S}$ (resp. $\beta=\alpha_{N}$ ) and $H=d m n \beta$. Suppose $d m n \beta_{U} \ni g_{n} \underset{n=1,2, \cdots}{ } g$ and $\sup _{n} \beta_{U}\left(g_{n}\right)$ exists in $Y$. Then $g \in d m n \beta_{U}$ and $\beta_{U}(g)=\sup _{n} \beta_{U}\left(g_{n}\right)$.

Proof. By replacing $\left\{g_{n}\right\}$ if necessary by $\left\{g_{n}-g_{1}\right\}$ we can assume that $g_{1} \geqq 0$. In light of Theorems 2.2 and 4.2 we need only show that $g \in U \cap d m n \underline{\beta}$ and $\underline{\beta}(g)=\sup _{n} \beta_{U}\left(g_{n}\right)$. Given any $h$ in $d m n \beta$ it is clear that $g_{n} \wedge h \underset{n=1,2, \ldots}{\uparrow} g \wedge h$. By the previous theorem $\bar{\beta}(g \wedge h)=$ $\sup _{n} \bar{\beta}\left(g_{n} \wedge h\right)=\sup _{n} \beta_{U}\left(g_{n} \wedge h\right) \leqq \sup _{n} \beta_{U}\left(g_{n}\right)$. Since $\sup _{n} \beta_{U}\left(g_{n} \wedge h\right)=$ $\sup _{n} \underline{\beta}\left(g_{n} \wedge h\right) \leqq \underline{\beta}(g \wedge h)$ we conclude that $\bar{\beta}(g \wedge h)=\underline{\beta}(g \cdot h)$. Thus 
$g \wedge h \in d m n \beta$ for all $h \in d m n \beta$ by Theorem 3.2, and $\beta(g \wedge h) \leqq$ $\sup _{n} \beta_{U}\left(g_{n}\right)$. This shows that $g \in U \cap d m n \underline{\beta}$ and then we conclude by Theorem 4.2 that $g \in d m n \beta_{U}$ and $\beta_{U}(g)=\beta(g)=\sup _{n} \beta_{U}\left(g_{n}\right)$.

In addition to this monotone convergence theorem we can prove, with the same hypotheses, the corresponding generalizations of Fatou's lemma and the dominated convergence theorem. We use the standard vector lattice definitions of $\overline{\mathrm{lim}}$ and $\mathrm{lim}$ and order limit and the proofs follow from the monotone convergence theorem above just as they do in the chapter on the Daniell integral in [9].

Given a subspace $H$ of a vector lattice $V$ we define $\sigma(H)$ to be the set of $f \in V$ such that $|f|=\sup _{n}\left(|f| \wedge h_{n}\right)$ for some sequence $\left\{h_{n}\right\}$ in $H$. If $\alpha_{S}$ is the sequential extension of a Daniell function $\alpha$, then we call the restriction of $\alpha_{S U}$ to $\sigma\left(d m n \alpha_{S}\right)$ the Daniell extension of $\alpha$ and denote it by $\alpha_{D}$. If $\alpha$ has the nonsequential Daniell property then we denote $\alpha_{N U}$ restricted to $\sigma\left(d m n \alpha_{N}\right)$ by $\alpha_{B}$ and call it the Bourbaki extension of $\alpha$.

It is easy to see that $\sigma(H)$, for any $H$, is closed under countable sups and infs and sequential order limits. From this fact it is obvious that the convergence theorems of this section still hold for the Daniell and Bourbaki extensions. These extensions can also be characterized in another way. From the monotone convergence theorem it is easy to see that $\alpha_{S}$ and $\alpha_{N}$ are Daniell functions. If we take the sequential extensions of these functions we can prove without much difficulty that we obtain just the Daniell and Bourbaki extensions we have just defined. Finally the monotone convergence theorem shows that we get nothing new if we attempt to take yet another sequential extension.

7. Relations with Bourbaki and Daniell integrals. In [2] integration is defined in the following situation. Let $X$ be a locally compact Hausdorff space, let $V$ be the space of real-valued functions on $X$, and let $G=C_{00}(X)$, the space of continuous functions defined on $X$ having compact support. If $\alpha$ is a positive linear functional defined on $G$ then an extension $I$, of $\alpha$, is defined. This extension has the property that $I(f)=\underline{\alpha}(f)$ for all $f \in N(G)$. (In this situation the functions in $N$ are lower semicontinuous as can easily be seen. They do not include all lower semicontinuous functions, however, since they are bounded below and assume only finite values.) Dini's theorem shows that $\alpha$ has the nonsequential Daniell property so it is possible to define $\alpha_{N}, \alpha_{B}$, and $\alpha_{N U}$.

THEOREM 7.1. Let $X$ be a locally compact Hausdorff space and let $V$ be the space of all real-valued functions on $X$. Suppose that $G$ is the space of continuous real-valued functions on $X$ with compact support 
and let $\alpha$ be any positive linear functional on $G$. Then $\alpha_{B}$ is the integral I, of Bourbaki, restricted to finite-valued functions.

Proof. Let $f$ be in $d m n \alpha_{B}$. Since $d m n \alpha_{B}$ is a sublattice we can assume that $f$ is positive. There exists a sequence $\left\{g_{n}\right\}$ in $d m n \alpha_{N}$ such that $f=\sup \left\{f \wedge g_{n}: n=1,2, \cdots\right\}$ and $\sup _{n} \alpha_{N}\left(f \wedge g_{n}\right)=\alpha_{N}(f)$. But for each $n$ we have $\alpha_{N}\left(f \wedge g_{n}\right)=\inf \left\{\underline{\alpha}(h): f \wedge g_{n} \leqq h \in N\right\}=$ $\sup \left\{\bar{\alpha}(p): f \wedge g_{n} \geqq p \in-N\right\}$ by $N$-approximation and the fact that $\alpha_{N}=\underline{\alpha}$ on $N$. Hence by [2; p. 151] we see that each $f \wedge g_{n}$ is integrable in the sense of Bourbaki and $I\left(f \wedge g_{n}\right)=\alpha_{N}\left(f \wedge g_{n}\right)$. Then the monotone convergence theorem for the Bourbaki integral shows that $f \in d m n I$ and $I(f)=\alpha_{N}(f)$.

Conversely if $f$ is a real-valued integrable function we assume first that $f$ is positive just as we did above. By [2; p. 151] for any $\epsilon>0$ we can assume that there is $g \geqq 0$ in $-N$ and $h$ which is lower semicontinuous and integrable (but not necessarily in $N$ since it may assume infinite values) such that $g \leqq f \leqq h$ and $I(h-g)<\epsilon$. Now given any positive integer $n$ we have $g \wedge n \leqq f \wedge n \leqq h \wedge n$ and $I((h \wedge n)-(g \wedge n))<\epsilon$. Then $h \wedge n$ belongs to $N$ and the fact that $h \wedge n$ is integrable implies that $I(h \wedge n)=\underline{\alpha}(h \wedge n)=$ $\alpha_{N}(h \wedge n)$. Similarly $I(g \wedge n)=\alpha_{N}(g \wedge n)$. We conclude that $\left(\underline{\alpha_{N}}\right)(f \wedge n)=\overline{\left(\alpha_{N}\right)}(f \wedge n)$ which means that $f \wedge n$ is in $d m n \alpha_{N}$ by Theorem 3.2. It is then clear that $\alpha_{N}(f \wedge n)=I(f \wedge n)$ for all $n$. Since $f=\sup _{n}(f \wedge n)$ the monotone convergence theorem for $\alpha_{B}$ gives the result.

Bourbaki extends the integral $I$ to an essential integral $J$ in the following way. In [2; p. 183] we see that a set $X$ is locally of measure zero if its intersection with every compact set is of measure zero. Then in $[3 ;$ p. 13] we see that a function $f$ is essentially integrable if and only if it can be written in the form $f=g+h$ where $g$ is integrable and $h$ is such that $\{x \in X: h(x) \neq 0\}$ is locally of measure zero. Under the assumptions of the previous theorem it can be shown that $\alpha_{N U}$ is the essential integral.

Now consider the case when $V$ is the space of real-valued functions defined on a set $X$. Let $G$ be a vector sublattice of $V$ such that $f \wedge c$ and $f \vee(-c)$ are in $G$ for all $f$ in $G$ and all positive real numbers $c$. Let $\alpha$ be a positive linear functional defined on $G$ with the Daniell property. The Daniell integral is defined by Segal and Kunze in [10] as follows. Let $S^{*}$ be the set of all extended-real-valued functions $f$ such that $f$ is the sup of a sequence of functions from $G$. Now define $G^{*}$ to be the set of all $f$ in $V$ such that inf $\alpha\left(\left(f+V^{+}\right) \cap\left(S^{*}\right)\right)=$ $\sup \bar{\alpha}\left(\left(f-V^{+}\right) \cap\left(-S^{*}\right)\right)=($ a finite value). Then the Daniell integral of $f, I(f)$, is equal to this value for any $f$ in $G^{*}$. 
THEOREM 7.3. Let $V$ be the space of real-valued functions defined on a set $X$. Let $G$ be a vector sublattice of $V$ such that $f \wedge c$ and $f \vee(-c)$ are in $G$ for all $f$ in $G$ and all positive real numbers $c$. Let $\alpha$ be $a$ positive linear functional defined on $G$ with the Daniell property. Then $\alpha_{D}$ is the Daniell integral as defined above.

Proof. Let $f$ be in $d m n \alpha_{D}$ and suppose as usual that $f \geqq 0$. Then by definition there is a sequence $\left\{h_{n}\right\}$ in $d m n \alpha_{S}$ such that $f \wedge h_{n}$ is in $d m n \alpha_{S}$ for all $n$ and $f=\sup _{n} f \wedge h_{n}$. By $S$-approximation $\alpha_{S}\left(f \wedge h_{n}\right)=$ inf $\underline{\alpha}\left(\left(f \wedge h_{n}+V^{+}\right) \cap(S)\right)=\sup \bar{\alpha}\left(\left(f \wedge h_{n}-V^{+}\right) \cap(-S)\right)$ so we conclude that $f \wedge h_{n}$ is in $G^{*}$ for all $n$ and $I\left(f \wedge h_{n}\right)=\alpha_{S}\left(f \wedge h_{n}\right)$. But then the monotone convergence theorems for $\alpha_{D}$ and the Daniell integral show that $f$ is in $G^{*}$ and $\alpha_{D}(f)=I(f)$.

If $f$ is in $G^{*}$ and $f \geqq 0$ then the condition on $G$ allows us to say that $f \wedge n$ is in $G^{*}$ for all positive integers $n$. Since $f \wedge n$ is bounded it is easy to see that $f \wedge n$ is in $d m n \alpha_{s}$ for all $n$ and $I(f \wedge n)=$ $\alpha_{S}(f \wedge n)$. Because $f=\sup _{n} f \wedge n$ the monotone convergence theorems for $\alpha_{D}$ and for $I$ give the result.

In a fashion analogous to the procedure in the definition of the essential integral we can define a set $A$ to be of local measure zero if the characteristic function of $A \cap E$ has integral zero for every set $E$ with integrable characteristic function. A function $f$ is said to be zero locally almost everywhere if $\{x: f(x) \neq 0\}$ is a set of local measure zero. A function $f$ is said to be extendedly integrable with integral $J(f)$ if there is an integrable function $g$ such that $f=g$ locally almost everywhere and then $J(f)$ is defined to be $I(g)$. It can be shown that this extended Daniell integral [10] restricted to finite-valued functions is just $\alpha_{S U}$.

\section{The Choquet-Bishop-de Leeuw theorem in vector} lattices. In this section we will apply our previous results to a proof of a generalization of the Choquet-Bishop-de Leeuw theorem on integral representations.

Let $V$ be a vector lattice and let $G$ be a subspace of $V$ and let $\alpha$ be a real-valued positive linear function defined on $G$. To use the theory of previous sections we need a positive linear function defined on a sublattice of $V$. Thus it is natural to first extend $\alpha$ to a monotonic linear functional $\hat{\alpha}$ defined on the smallest vector sublattice containing G.

Let $C$ be the wedge of finite infs from $G$. Then it is clear that $C-C$ is a subspace contained in all vector sublattices containing ป. But if $a, b \in C$ then $(a-b)^{+}=a-(a \wedge b)$, an element of $C-C$, so we see that $C-C$ is the smallest vector sublattice containing $G$. Since 
$C$ is a wedge we can apply our basic theorem and take a $C$-maximal extension $\hat{\alpha}$ of $\alpha$. If $C \subset\left(G+V^{+}\right) \cap\left(G-V^{+}\right)$then $d m n \hat{\alpha} \supset C-C$ for all positive linear functionals $\alpha$. If $G \subset G^{+}-G^{+}$it is easy to see that $C \subset\left(G+V^{+}\right) \cap\left(G-V^{+}\right)$so we will assume for the remainder of this section that $G \subset G^{+}-G^{+}$. If $\alpha$ has the Daniell property we can consider $(\hat{\alpha})_{D}$ and this will be the generalization of the measure whose existence is asserted in the Choquet-Bishop-de Leeuw theorem.

Let $X$ be a compact Hausdorff space, let $V=R^{X}$, and let $G$ be a subspace of $C(X)$ which contains constants and separates points of $X$. We define the Choquet boundary corresponding to $G$ to be the set $\partial G$ of elements of $X$ with the property that the corresponding linear functional $\epsilon_{x}: G \rightarrow R$ defined by $\epsilon_{x}(f)=f(x)$ has a unique positive linear extension to $C(X)$. If we let $W$ be the wedge of increasing sequential sups from the wedge $C$ of finite infs from $G$ then Bauer's minimum principle [8, p. 225] shows that, if $f \in W$ and $f(x)>0$ for all $x \in \partial G$, then $f(x)>0$ for all $x \in X$.

Now if $\alpha$ is any positive linear functional on $G$ then, from the

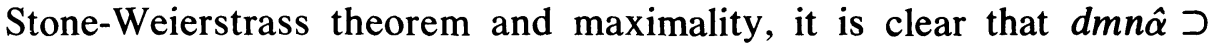
$C(X)$ for any maximal $C$-extension $\hat{\alpha}$ of $\alpha$. If we let $\beta$ be the restriction of $\hat{\alpha}$ to $C(X)$ then $\beta$ is $C$-approximated and $\beta$ is a Daniell function by Dini's theorem. If we can show that $\beta_{1}$ is $W$-approximated where $\beta_{1}$ is the restriction of $\beta_{D}$ to bounded functions, then the Choquet-Bishop-de Leeuw theorem will be proved. Indeed, if $f$ is the characteristic function of a Baire set disjoint from $\partial G$ and if $f \geqq$ $g \in-W$, then Bauer's minimum principle shows that $g \leqq 0$. Since $f$ is a Baire function it is integrable by any measure so $\beta_{1}$ is defined on $f$ and then $W$-approximation gives $\beta_{1}(f)=\sup \beta_{1}\left(\left(f-V^{+}\right) \cap(-W)\right) \leqq 0$ so $\beta_{1}(f)=0$. We conclude that any positive linear functional on $G$ can be extended to a measure which assigns measure zero to any Baire set disjoint from $\partial G$. From the following theorem it will be clear that $\beta_{1}$ is $W$-approximated.

THEOREM 8.1. Let $H$ be a sublattice of a Dedekind $\sigma$-complete vector lattice $V$ and suppose that $\alpha$ is a positive linear functional on $H$ with the Daniell property. Let $W$ be a wedge in $S(H)$ such that $\alpha$ is $W$-approximated and such that $W$ is closed under increasing sequential sups and finite infs. Let $\beta$ be the restriction of $\alpha_{D}$ to $\left(W-V^{+}\right) \cap$ $\left(-W+V^{+}\right)$. Then $\beta$ is $W$-approximated.

Proof. First consider $f \in(-S) \cap(d m n \beta)$. We adapt a technique due to Choquet $[8 ; \quad$ p. 233] to show that $\beta(f)=$ $\sup \beta\left(\left(f-V^{+}\right) \cap(-W)\right.$. Since $f$ is in $d m n \beta$ there is $h \in-W$ such that $h \leqq f$. Because $f \in(-S)$ there is a sequence $\left\{f_{n}\right\}$ of elements of $H$ such that $f_{n} \underset{n=1,2, \cdots}{\downarrow} f$ and $\alpha\left(f_{n}\right) \underset{n=1,2, \cdots}{\downarrow} \beta(f)$. Choose $g_{1} \in(-W) \cap H$ such 
that $g_{1} \leqq f_{1}$ while $\alpha\left(g_{1}\right) \geqq \alpha\left(f_{1}\right)-\frac{1}{2} \epsilon$. We inductively define $g_{n} \in$ $(-W) \cap H$ such that $g_{n} \leqq g_{n-1} \wedge f_{n}$ while $\alpha\left(g_{n}\right) \geqq \alpha\left(f_{n} \wedge g_{n-1}\right)-$ $\epsilon \cdot 2^{-n}$. Since $-W$ is closed under finite sups we can assume $g_{n} \geqq h$ for all $n$ by taking $g_{n} \vee h$ if necessary. Then:

$$
\begin{aligned}
\alpha\left(f_{n}-g_{n}\right) & =\alpha\left(f_{n}-\left(f_{n} \wedge g_{n-1}\right)\right)+\alpha\left(\left(f_{n} \wedge g_{n-1}\right)-g_{n}\right) \\
& \leqq \alpha\left(f_{n-1}-g_{n-1}\right)+\epsilon \cdot 2^{-n} .
\end{aligned}
$$

Adding, we find that $\alpha\left(f_{n}-g_{n}\right) \leqq \epsilon$ for all $n$. Let $g=\inf _{n} g_{n} \geqq$ $h$. Then $g \in-W$ since the $g_{n}$ 's form a decreasing sequence and Theorem 6.2 shows that $g$ is in $d m n \beta$. Clearly $f \geqq g$ while $\beta(g)=$ $\inf _{n} \alpha\left(g_{n}\right) \geqq \beta(f)-\epsilon$. This shows that $\beta(f)=\sup \beta\left(\left(f-V^{+}\right) \cap(-W)\right.$.

Now assume that $f$ is any element of $d m n \beta$. If $f \geqq$ $g \in(-S) \cap d m n \alpha_{D}$ choose $h \in-W$ such that $f \geqq h$. Then $f \geqq g \vee h$ and it is easy to see that $g \vee h \in(-S) \cap(d m n \beta)$. Hence $\alpha_{D}(g) \leqq$ $\beta(g \vee h)=\sup \beta\left(\left(g \vee h-V^{+}\right) \cap(-W)\right)$. Thus

$$
\beta(f) \geqq \sup \beta\left(\left(f-V^{+}\right) \cap(-W)\right) \geqq \sup \alpha_{D}\left(\left(f-V^{+}\right) \cap(-S)\right)=\beta(f) .
$$

We conclude that $\beta$ is $W$-approximated.

9. Vector integrals. In this section we indicate how the methods of previous sections may be applied to define integrals with values in locally convex spaces. Let $Y$ be a vector space with a locally convex topology determined by a family $P$ of seminorms. Changing slightly the method used by DeMarr in [5], we define $P(Y)$ to be the product space $R^{P} \times Y$ with a relation “ $\leqq$ " defined by:

$$
\begin{gathered}
\left(h_{1}, y_{1}\right) \leqq\left(h_{2}, y_{2}\right) \text { if and only if } p\left(y_{2}-y_{1}\right) \leqq h_{2}(p)-h_{1}(p) \\
\text { for all } p \in P .
\end{gathered}
$$

THEOREM 9.1. The space $P(Y)$ with relation "@" is a preordered vector space which is antisymmetric if and only if $Y$ is Hausdorff. Given $p \in P$, if we define $f_{p}$ on $P(Y)$ by $f_{p}(h, y)=h(p)$, then $f_{p}$ is a nonsequential Daniell function.

Proof. The first statement is easily verified. As for the second, given $p \in P$, if we define $f_{p}$ by $f_{p}(h, y)=h(p)$, then it is clear that $f_{p}$ is a monotonic linear functional. Suppose $\left\{\left(h_{a}, y_{a}\right): a \in A\right\}$ is a decreasing lower bounded family in $P(Y)$ with only negative lower bounds. Let $(g, z)$ be a lower bound for the family. Since $\left\{h_{a}(p): a \in A\right\}$ is lower bounded by $g(p)$ we see that the family decreases to an infimum $m(p)$ for each $p$ in $P$. Suppose there exists $q$ in $P$ such that $m(q)>$ 
0 . Then if we choose $b$ in $A$ such that $h_{b}(q)<(3 / 2) m(q)$ and define the function $n$ by; $n(q)=\frac{1}{2} m(q)$ and $n(p)=2 m(p)-h_{b}(p)$ for $p \neq q$ we will contradict the assumption $m(q)>0$ by showing that $\left(n, y_{b}\right)$ is a nonnegative lower bound for $\left\{\left(h_{a}, y_{a}\right): a \in A\right\}$.

Given any $a$ in $A$ such that $\left(h_{a}, y_{a}\right) \leqq\left(h_{b}, y_{b}\right)$ and any $p$ in $P$ different from $q$ we have;

$$
\begin{aligned}
p\left(y_{a}-y_{b}\right) & =p\left(y_{b}-y_{a}\right) \leqq h_{b}(p)-h_{a}(p) \leqq h_{b}(p)-m(p) \\
& \leqq\left(h_{b}(p)+\left(h_{a}(p)-m(p)\right)\right)-m(p)=h_{a}(p)-n(p),
\end{aligned}
$$

while

$$
q\left(y_{a}-y_{b}\right)=q\left(y_{b}-y_{a}\right) \leqq h_{b}(q)-h_{a}(q) \leqq \frac{1}{2} m(q) \leqq h_{a}(q)-n(q)
$$

since $h_{b}(q)$ and $h_{a}(q)$ are both in $[m(q),(3 / 2) m(q)]$. Thus $\left(n, y_{b}\right) \leqq$ $\left(h_{a}, y_{a}\right)$ and since we are dealing with a decreasingly directed family we conclude that $\left(n, y_{b}\right)$ is the required nonnegative lower bound.

THEOREM 9.2. If $Y$ is a quasi-complete locally convex space (i.e., $Y$ is Hausdorff and every closed, topologically bounded subset of $Y$ is complete) then $P(Y)$ is a $Q$-space.

Proof. We first show that $P(Y)$ is monotone Dedekind complete. Let $\left\{\left(h_{a}, y_{a}\right): a \in A\right\}$ be an increasingly directed family, upper bounded by an element $(g, z)$. Since the family is increasingly directed we can assume that $\left(h_{a}, y_{a}\right) \geqq\left(g_{1}, z_{1}\right)$ for all $a \in A$. Then for any $p \in P$ we have:

$$
\begin{aligned}
p\left(y_{a}\right) & \leqq p\left(z-y_{a}\right)+p(z) \leqq g(p)-h_{a}(p)+p(z) \\
& \leqq g(p)-g_{1}(p)+p(z)
\end{aligned}
$$

so the family is topologically bounded. Let $f \in R^{P}$ be defined by $f(p)=\sup _{a} h_{a}(p)$. Given any $p \in P$ and $\epsilon>0$ choose $b \in A$ such that $f(p)-h_{b}(p)<\epsilon$. Then for any $a \in A$ such that $\left(h_{a}, y_{a}\right) \geqq\left(h_{b}, y_{b}\right)$ we have $p\left(y_{a}-y_{b}\right) \leqq h_{a}(p)-h_{b}(p)<\epsilon$. Thus the family $\left\{y_{a}: a \in A\right\}$ is a Cauchy net and since it is topologically bounded we can find a limit $y \in Y$. Then $(f, y)$ is the least upper bound of $\left\{\left(h_{a}, y_{a}\right): a \in\right.$ $A\}$. Indeed, for any $p \in P$ and $a \in A$ we have, for any $b \in A$ such that $\left(h_{b}, y_{b}\right) \geqq\left(h_{a}, y_{a}\right)$ :

$$
h_{b}(p)-h_{a}(p) \geqq p\left(y_{b}-y_{a}\right) .
$$

If we take the limit over $b$ we find that $f(p)-h_{a}(p) \geqq p\left(y-y_{a}\right)$ so $(f, y)$ is an upper bound. A similar limit argument shows that it is the least upper bound. 
To complete the proof we need to demonstrate the existence of a separating family of nonsequential Daniell functionals. Given any $(h, y) \in P(Y)$ if $(h, y) \neq 0$ then either there is $p_{0} \in P$ such that $h\left(p_{0}\right) \neq 0$ or else $h=0$ and $y \neq 0$. In the first case we see that the linear functional $f_{p o}$ defined in the previous theorem sends $(h, y)$ to a nonzero value. If $h=0$ we note that there must be a $p \in P$ such that $p(y) \neq 0$. Let $g$ be a linear functional on $Y$ which is dominated by $p$ and which sends $y$ to a nonzero value. Then if we define $G_{p}$ on $P(Y)$ by $G_{p}(k, x)=k(p)-g(x)$ it is easy to see that $G_{p}$ is a positive linear functional. Since $G_{p} \leqq 2 f_{p}$ we see that $G_{p}$ is a nonsequential Daniell function. Finally $G_{p}(h, y)=-g(y) \neq 0$ gives the result.

THEOREM 9.3. Let $\alpha$ be a continuous linear function from $C_{00}(X)$, $X$ a locally compact Hausdorff space, to $Y, Y$ as in 9.2. Then there exists a Daniell function $\alpha \#: C_{00}(X) \rightarrow P(Y)$ such that $\alpha \#(x)=$ ( $h, \alpha(x)$ ) for each $x \in C_{00}(X)$, if and only if $\alpha$ has the following property:

(I) For each $p \in P$ there exists a positive linear functional $\beta_{p}$ on $C_{00}(X)$ such that $p(\alpha(x)) \leqq \beta_{p}(x)$ for all $x \in C_{00}(X)^{+}$.

Proof. If $\alpha \#$ exists then it is clear that, if we define $\beta_{p}$ on $C_{00}(X)$ by $\beta_{p}(x)=f_{p}(\alpha \#(x))$, then for $x \in C_{00}(X)^{+}$we have $\beta_{p}(x)=$ $f_{p}(\alpha \#(x)) \geqq p(\alpha(x))$.

Conversely if such a $\beta_{p}$ exists for each $p \in P$ we define $\alpha \#(x)=$ $(h, \alpha(x))$ where $h(p)=\beta_{p}(x)$ for all $x \in X$. Then it is clear that $\alpha \#$ is monotonic and linear and the Daniell property follows from Dini's theorem.

Now suppose that we are given a continuous linear function $\alpha$ from $C_{00}(X)$ to a quasi-complete locally convex space $Y$. If $\alpha$ has property (I) of the previous theorem (note that this property appears in [4]) then we can define the mapping $\alpha \#$ and can consider $(\alpha \#)_{D}$. It is easy to see that this mapping will possess all the convergence properties mentioned in $\S 6$. If we let $\alpha_{1}=p r_{2} \circ(\alpha \#)_{D}$ then $\alpha_{1}$ is a vector-valued integral which extends $\alpha$ and which has the property that if $\left\{x_{n}\right\}$ is a sequence in $d m n \alpha_{1},\left|x_{n}\right| \leqq y \in d m n \alpha_{1}$ and $x_{n} \rightarrow x$ then $x \in d m n \alpha_{1}$ and $\alpha_{1}(x)=\lim _{n} \alpha_{1}\left(x_{n}\right)$.

\section{REFERENCES}

1. E. M. Alfsen, Order Theoretic Foundations of Integration. Math. Ann., 149 (1963), 419-461.

2. N. Bourbaki, Integration, Ch. I-IV, Elem. de Math. Livre VI, Act. Sci. et Ind., No. 1175, Hermann et Cie, Paris (1952).

3. - Integration, Ch. V, Elem. de Math. Livre VI, Act. Sci. et Ind., No. 1244, Hermann et Cie, Paris (1956).

4. Integration, Ch. VI, Elem. de Math. Livre VI, Act. Sci. et Ind., No. 1281, Hermann et Cie, Paris (1959). 
5. R. DeMarr, Partially Ordered Linear Spaces and Locally Convex Linear Topological Spaces. Illinois J. Math., 8, No. 4, (1964), 601-606.

6. J. Kelley and I. Namioka, Linear Topological Spaces. D. Van Nostrand Co. Inc., Princeton, N. J. (1963).

7. E. McShane, Order-preserving Maps and Integration Processes. Annals of Math. Study No. 31 , Princeton Univ. Press, Princeton, New Jersey.

8. P. A. Meyer, Probability and Potentials. Blaisdell, Waltham, Mass. (1966).

9. H. Royden, Real Analysis, MacMillan, New York (1968).

10. I. Segal, and R. Kunze, Integrals and Operators, McGraw-Hill, New York (1968).

Received February 1, 1974.

UNIVERSITY OF NEW MEXICO 
D. E. Bennett, Strongly unicoherent continua ............................. 1

Walter R. Bloom, Sets of p-spectral synthesis ................................ 7

R. T. Bumby and D. E. Dobbs, Amitsur cohomology of quadratic extensions: Formulas and number-theoretic examples ................. 21

W. W. Comfort, Compactness-like properties for generalized weak topological sums

D. R. Dunninger and J. Locker, Monotone operators and nonlinear biharmonic boundary value problems ...

T. S. Erickson, W. S. Martindale, 3rd and J. M. Osborn, Prime nonassociative algebras

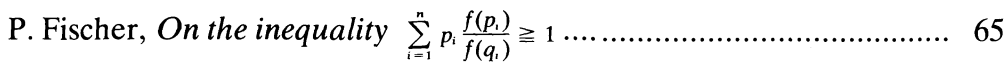

G. Fox and P. Morales, Compact subsets of a Tychonoff set ............... 75

R. Gilmer and J. F. Hoffmann, A characterization of Prüfer domains in terms of polynomials ......................................................... 81

L. C. Glaser, On tame Cantor sets in spheres having the same projection in each direction ......................................................... 87

Z. Goseki, On semigroups in which $X=X Y X=X Z X$ if and only if

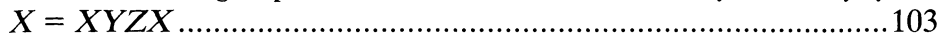

E. Grosswald, Rational valued series of exponentials and divisor

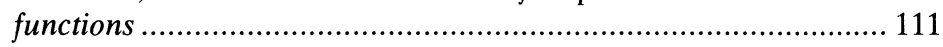

D. Handelman, Strongly semiprime rings ...................................... 115

J. N. Henry and D. C. Taylor, The $\bar{\beta}$ topology for $w^{*}$-algebras ............. 123

M. J. Hodel, Enumeration of weighted p-line arrays .......................... 141

S. K. Jain and S. Singh, Rings with quasiprojective left ideals .............. 169

S. Jeyaratnam, The diophantine equation $Y(Y+m)(Y+2 m) \times$

$$
(Y+3 m)=2 X(X+m)(X+2 m)(X+3 m) \ldots \ldots \ldots \ldots \ldots \ldots \ldots . . .183
$$

$\mathrm{R}$. Kane, On loop spaces without $p$ torsion .........................................189

Alvin J. Kay, Nonlinear integral equations and product integrals ..........203

A. S. Kechris, Countable ordinals and the analytic hierarchy, I ...........223

Ka-Sing Lau, A representation theorem for isometries of $C(X, E) \ldots \ldots . .229$

I. Madsen, On the action of the Dyer-Lashof algebra in $H_{*}(G)$..........235

R. C. Metzler, Positive linear functions, integration, and Choquet's theorem ........................................................................................ 277

A. Nobile, Some properties of the Nash blowing-up ............................297

G. E. Petersen and G. V. Welland, Plessner's theorem for Riesz conjugates 


\section{Pacific Journal of Mathematics}

\section{Vol. 60, No. $1 \quad$ September, 1975}

Donald Earl Bennett, Strongly unicoherent continua ................ 1

Walter Russell Bloom, Sets of p-spectral synthesis ................ 7

Richard Thomas Bumby and David Earl Dobbs, Amitsur cohomology of

quadratic extensions: formulas and number-theoretic examples .......

W. Wistar (William) Comfort, Compactness-like properties for generalized

weak topological sums ...................................

Dennis Robert Dunninger and John Stewart Locker, Monotone operators

and nonlinear biharmonic boundary value problems ..............

Theodore Erickson, Wallace Smith Martindale, III and J. Marshall Osborn,

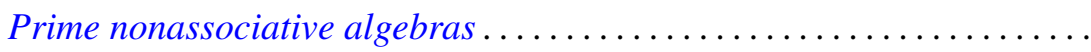

Pál Fischer, On the inequality $\sum_{i=0}^{n}\left[f\left(p_{i}\right) / f\left(q_{i}\right)\right] p_{i} \geq i \ldots \ldots \ldots \ldots \ldots$

Geoffrey Fox and Pedro Morales, Compact subsets of a Tychonoff set.......

Robert William Gilmer, Jr. and Joseph F. Hoffmann, A characterization of

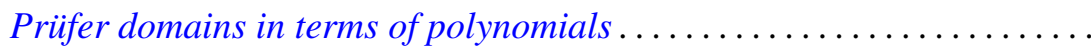

Leslie C. Glaser, On tame Cantor sets in spheres having the same projection

in each direction . ...................................

Zensiro Goseki, On semigroups in which $x=x y x=x z x$ if and only if

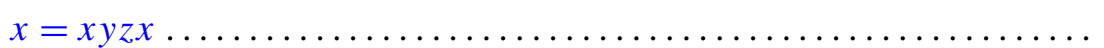

Emil Grosswald, Rational valued series of exponentials and divisor

functions.

David E. Handelman, Strongly semiprime rings

Jackson Neal Henry and Donald Curtis Taylor, The $\bar{\beta}$ topology for

$W^{*}$-algebras

Margaret Jones Hodel, Enumeration of weighted p-line arrays ...

Surender Kumar Jain and Surjeet Singh, Rings with quasi-projective left

ideals.

S. Jeyaratnam, The Diophantine equation

$$
Y(Y+m)(Y+2 m)(Y+3 m)=2 X(X+m)(X+2 m)(X+3 m) \ldots
$$

Richard Michael Kane, On loop spaces without $p$ torsion

Alvin John Kay, Nonlinear integral equations and product integrals ...

Alexander S. Kechris, Countable ordinals and the analytical hierarchy.

$I$.

Ka-Sing Lau, A representation theorem for isometries of $C(X, E)$

Ib Henning Madsen, On the action of the Dyer-Lashof algebra in $H_{*}(G)$

Richard C. Metzler, Positive linear functions, integration, and Choquet's

theorem.

Augusto Nobile, Some properties of the Nash blowing-up

Gerald E. Peterson and Grant Welland, Plessner's theorem for Riesz. 